\title{
Visual and Anticipatory Bias in Three Cortical Eye Fields of the Monkey during an Adaptive Decision-Making Task
}

\author{
Brian Coe, Kazuya Tomihara, Masako Matsuzawa, and Okihide Hikosaka \\ Department of Physiology, School of Medicine, Juntendo University, 2-1-1 Hongo, Bunkyo-Ku, Tokyo 113, Japan
}

To examine the role of three cortical eye fields during internally guided decision-making processes, we recorded neuronal activities in the frontal eye field (FEF), supplementary eye field (SEF), and lateral intraparietal cortex (LIP) using a free-choice delayed saccade task with two synchronized targets. Although the monkeys must perform the task in a time-locked manner, they were free to choose either the receptive field (RF) target or the nonreceptive field (nRF) target to receive reward. In all three areas we found neurons with stronger activation during trials when the monkey was going to make a saccade to the RF target (RF trials) than to the $\mathrm{nRF}$ target (nRF trials). Modulation occurred not only during target presentation (visual bias) but also before target presentation (anticipatory bias). The visual bias was evident as an attenuated visual response to the RF stimulus in nRF trials. The anticipatory bias, however, was seen as an enhancement of pretarget activity in the RF trials. We analyzed the activity during the $500 \mathrm{msec}$ before target presentation and found that $22.5 \%$ of FEF and $31.3 \%$ of LIP neurons and $49.1 \%$ of SEF neurons showed higher activity during the RF trials. To more accurately determine when each neuron started to show preferential activity, we used a new inverse interspike interval analysis procedure. Our results suggest that although all three cortical eye fields reflect attentional and intentional aspects of sensorimotor processing, SEF plays an earlier and perhaps more cognitive role in internally guided decision-making processes for saccades.

Key words: frontal eye fields; supplementary eye fields; lateral intraparietal cortex; single-cell activity; attention; intention; decision making; free-choice task; saccade
Many areas of the primate brain contribute to the generation and control of saccadic eye movements. At least three areas in the monkey cerebral cortex are thought to participate actively in saccade initiation: the frontal eye field (FEF) (Bruce and Goldberg, 1985), the supplementary eye field (SEF) (Schlag and Schlag-Rey, 1987), and the lateral intraparietal cortex (LIP) (Andersen and Gnadt, 1989). Neurons in these areas are not only active before and during saccades but also respond to visual targets (Mohler et al., 1973; Goldberg and Bushnell, 1981; Schall, 1991b; Colby et al., 1996). All three of these cortical eye fields also project to the superior colliculus (SC) where saccadic or gaze commands are generated (Segraves and Goldberg, 1987; Shook et al., 1990; Paré and Wurtz, 1997; Sommer and Wurtz, 2000). These studies suggest that the cortical mechanism for visually guided saccade initiation is distributed among at least three cortical eye fields. Such a distributed and presumably parallel network seems to be a general feature of neuronal mechanisms underlying complex behaviors. However, this raises two important questions:

Received Dec. 20, 2001; revised Feb. 28, 2002; accepted March 25, 2002.

This work was supported by a grant-in-aid for Scientific Research on Priority Areas (C) from the Ministry of Education, Culture, Sports, Science, and Technology, by Core Research for Evolutional Science and Technology of Japan Science and Technology Corporation, and by Japan Society for the Promotion of Science Research for the Future program. We thank Johan Lauwereyns, Shunsuke Kobayashi, Hiro Nakahara, and Makoto Sato for helpful comments, Makoto Kato for aid in designing the online computer program, and Masashi Koizumi for technical support.

Correspondence should be addressed to Okihide Hikosaka, Laboratory of Sensorimotor Research, National Eye Institute, National Institutes of Health, Building 49, Room 2A50, Bethesda, MD 20892. E-mail: oh@1sr.nei.nih.gov.

K. Tomihara's present address: Department of Psychology, Faculty of Law, Economics, and Humanities, Kagoshima University, 1-21-30 Korimoto, Kagoshima 890-0065, Japan.

M. Matsuzawa's present address: Department of Psychology, Faculty of Letters, Showa Woman's University, 1-7 Taishido, Setagaya-Ku, Tokyo 154-8533, Japan.

Copyright (C) 2002 Society for Neuroscience $0270-6474 / 02 / 225081-10 \$ 15.00 / 0$
Why are there three cortical eye fields, and how do they cooperate or compete to make a saccade?

In most experimental situations to date, the subject's decision when and where to make a saccade was dictated explicitly by instruction and reward; therefore, the subject's performance was exogenously controlled and highly predictable. In our daily life, however, we can easily choose between competing objects or locations to select as a target for a saccade without explicit instruction, often relying on internal bias. For brevity, we refer to these two kinds of decision-making processes as "externally guided" and "internally guided" decisions, understanding that these are over-simplified terms. Externally guided decision, or discrimination, tasks have been well studied (Schall, 1995, 2001; Kim and Shadlen, 1999).

We speculated that clear differences between the neuronal activity of the three areas might become apparent when investigating internally guided decision-making processes using the same task. To test this hypothesis, we needed to create a paradigm that did not blatantly dictate behavior and discouraged purely random selection. We devised a task where two identical stimuli were displayed and subjects were free to make a saccade to either one of them to obtain reward. A key feature of our paradigm is that the amount of reward waxed and waned gradually. This reward schedule encouraged but did not instruct the monkeys to choose one target for several consecutive trials and then switch to the other target. In this way, the subject was still free to select either target, because it would always receive reward, but could ascertain, after investigation and observation, which target it preferred. This complex decision-making task we feel is best described by the oxymoron "free-choice (FC) task." Using this task, we found neurons in the three cortical eye fields, especially the SEF, with anticipatory activity before target pre- 
sentation that predicted the monkey's saccadic behavior. Some of the findings presented have been published previously in abstract form (Coe et al., 1998, 2001).

\section{MATERIALS AND METHODS}

\section{General}

We used five male Japanese monkeys (Macaca fuscata; subjects H, K, P, $\mathrm{L}$, and $\mathrm{C}$ ). The monkeys were kept in individual primate cages in an air-conditioned room with food available ad libitum. At the beginning of each experimental session, they were moved to the experimental room in a primate chair. The monkeys were given restricted amounts of fluid during periods of training and recording. Their body weight and appetite were checked daily. Supplementary water and fruit were provided daily. The experiments were performed while the monkey's head was fixed and its eye movements were recorded. For this purpose, we implanted a head holder, a chamber for unit recording, and an eye coil under general anesthesia (Nakamura et al., 1998). All surgical and experimental protocols were approved by the Juntendo University Animal Care and Use Committee and are in accordance with the National Institutes of Health Guide for the Care and Use of Laboratory Animals.

\section{Experimental procedures}

During the training and recording sessions, each monkey was seated in a primate chair and placed in a sound-attenuated and dimly lit room with its head fixed. Visual stimuli consisted of small red spots of light (diameter, $0.4^{\circ}$ ) and were back-projected, using light-emitting diode projectors, onto a tangent screen $25 \mathrm{~cm}$ in front of the monkey's face. Eye movements were recorded using the search coil method (Robinson, 1963). Each monkey's performance was reinforced after each successful trial by drops of water. Water was dispensed through a "double barrel" tube arrangement, where one tube led to the pipe supplying the water and the other was left open, preventing the monkeys from sucking excess water.

Before single-unit recording began, we obtained magnetic resonance (MR) images (0.3 T; Airis; Hitachi, Tokyo, Japan) perpendicular to each recording chamber. Based on multitiered surface MR images, we could readily determine the locations of the three cortical eye fields: (1) FEF (monkeys $\mathrm{P}, \mathrm{H}$, and $\mathrm{K}$ ), in and around the anterior bank of the arcuate sulcus (Bruce et al., 1985); (2) SEF (monkeys P, C, and L), in the dorsomedial convexity, $2-5 \mathrm{~mm}$ from the midline, slightly anterior to the level of FEF (Shook et al., 1990); and (3) LIP (monkey P), in the lateral bank of the intraparietal sulcus (Barash et al., 1991b).

Single-unit recording was then performed using tungsten electrodes (diameter, $0.25 \mathrm{~mm}$; 1-5 M ; measured at $1 \mathrm{kHz}$; Frederick Haer, Bowdoinham, ME). A hydraulic microdrive (MO95-S; Narishige, Tokyo, Japan) was used to advance electrodes into the brain. The locations of the cortical eye fields were reconfirmed by the presence of visually responsive cells and, for the FEF and SEF, electrically evoked saccades with thresholds of $<50 \mu \mathrm{A}$ (Bruce et al., 1985; Schlag and Schlag-Rey, 1987).

The receptive fields (RFs) of visually responsive neurons were elucidated using a memory-guided task (Hikosaka and Wurtz, 1983). Once neurons were properly isolated using a BAK dual-window discriminator (model DDIS-1; BAK Electronics, Germantown, MD), single-unit recording was performed during $\sim 100$ trials of the FC task and 60 trials of a similar control task.

\section{Task procedures}

\section{FC task}

The FC task is shown in Figure 1 (left). Each trial started with the appearance of a central spot of light on which the monkey had to fixate (Fig. 1A). After $1200 \mathrm{msec}$, two spots appeared simultaneously, one in the RF of the cell (RF target) and the other outside the RF [nonreceptive field (nRF) target] (Fig. 1B). During this time, the monkey had to maintain fixation for another $800 \mathrm{msec}$. This period is referred to as the "visual period," because both targets were visible but the monkey had to maintain fixation. The nRF target was placed diametrically opposed to the RF target with respect to the fixation point (Fig. 1, left). After the visual period of $800 \mathrm{msec}$, the central fixation point was turned off, signaling the monkey to make a saccade (Fig. 1C). The monkey could then make a saccade to either of the two targets to obtain reward. Reward consisted of water (released by a solenoid) for a maximum of 150 msec duration and a tone pulse. The duration of the tone pulse was fixed

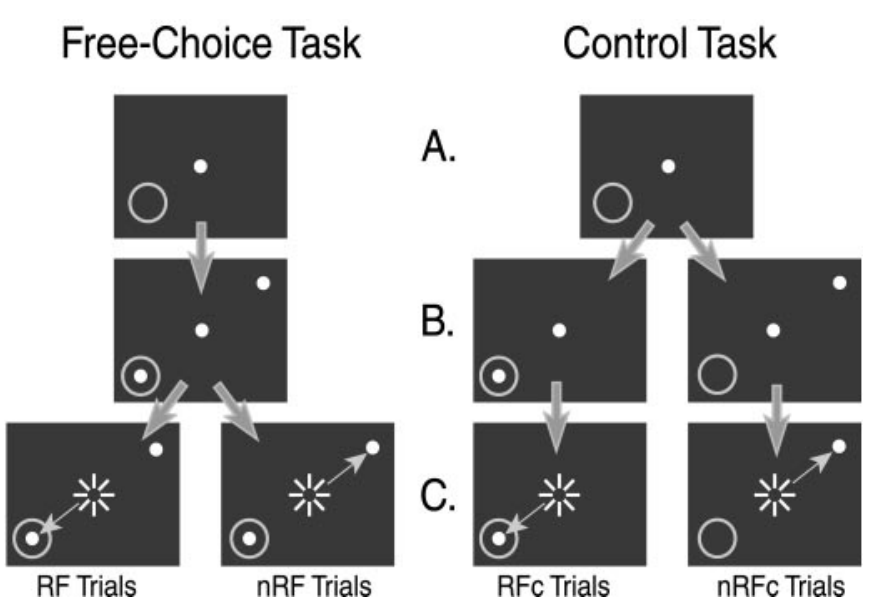

Figure 1. A schematic of the tasks. Left, The FC task. A, Fixation period. The subject must maintain fixation for $1200 \mathrm{msec}$. The gray ring indicates the RF of the cell. $B$, Visual period. Two targets come on but the subject must maintain fixation for another $800 \mathrm{msec}$. $C$, Saccade period. The fixation offset is the cue for the subject to make a saccade to the target inside the RF (RF target) or to the target outside the RF (nRF target). Successful trials in the FC task are categorized as RF trials and nRF trials based on the monkey's behavior. Right, The control task. A, Fixation period (same as the FC task). $B$, Visual period. Only one target is presented, and the subject must still maintain fixation for another 800 msec. $C$, Saccade period. The fixation offset is the cue for the subject to make a saccade to the target presented. Successful trials in the control task are referred to as RFc trials and $\mathrm{nRFc}$ trials based on the target presented. The white dots represent the visual stimuli and are the only items visible to the subject. Large arrows indicate correct trial progression. Small arrows indicate saccades made. Sunbursts indicate fixation offset.

at $75 \mathrm{msec}$ so that the only clue as to the duration of the reward was the amount of water dispensed.

To encourage the monkey to participate more actively in the task, the computer program adjusted the amount of reward after most trials using several guidelines, as shown below. However complex these "policies" may appear, the monkey was always free to choose either one of the targets to receive reward. The reward scheme was designed to react to, not instruct, the monkey.

Independence policy. Each target had its own independent reward amount. The reward amount for either target would only change if it had been chosen (but see Bonus policy, below).

Initialization policy. At the start of each block of trials, the reward amount for both targets is set to $30 \%$ of maximum. A saccade to either target would be rewarded by the release of the current amount of reward for the target chosen and then the reward amount for the chosen target would increase by $10 \%$ for the next trial. The first target to be chosen three times would be designated the incrementing target and the other target would be designated the decrementing target. This nomenclature was set regardless of the spatial attributes of each target and was based solely on the monkey's behavior.

Main policy. Once the initialization was complete, a saccade to the incrementing target would dispense the current amount of reward for that target and then increase the amount of reward for that target by $10 \%$ for the next trial until it reached $100 \%(150 \mathrm{msec})$. A saccade to the decrementing target would dispense the current amount of reward and then decrease the reward amount for that target by $10 \%$ for the next trial until it reached $20 \%(30 \mathrm{msec})$.

Exchange policy. The reward amount for the incrementing target would remain at $100 \%$ for four to seven trials, randomly, to assure that the monkey would not know when the reward was going to start to decrease. At that point the designations would switch so that the previous incrementing target was now the decrementing target and vice versa.

Bonus policy. After an exchange, if the reward amount for the incrementing target was low $(\leq 40 \%)$, a small bonus of $20 \%$ would be added to it.

\section{Control task}

The control task is shown in Figure 1 (right). The time schedule of the control task was the same as the FC task, but only one target was 
presented for each trial and reward was always 50\% of maximum (75 $\mathrm{msec}$ ). The stimuli were identical to the ones used for the FC task but were presented in alternation. We avoided a random presentation schedule for the control task to prevent confounds that may have taken place by comparing the very predictable FC task with a nonpredictable control task.

\section{Data analysis}

Our initial interest for this study was in the modulation of visual responses in the three cortical areas, so purely saccadic cells or pause cells were not included in this data analysis.

\section{Context dependency}

For the data obtained from each neuron, we investigated the response of each cell in four conditions (Fig. 1): trials in the control task were classified into RF control (RFc) trials (saccades to the RF target) and $\mathrm{nRF}$ control (nRFc) trials (saccades to the $\mathrm{nRF}$ target); trials in the FC task were classified into RF trials (saccades to the RF target) and nRF trials (saccades to the nRF target). The RFc trials were used to set a standard for the response of the cell to a single target in its RF to which the monkey will make a saccade. The nRFc trials were used to set a standard for the response of the cell to a single saccade target diametrically opposed to the RF of the cell. The RF trials were used to gauge the response of the cell to having a second target outside the RF of the cell but still making a saccade to the RF target. The nRF trials were used to see how the cell would react when there was a target in its RF but the monkey had chosen to saccade to the nRF target instead.

Operationally we defined two kinds of context-dependent influences: (1) externally guided (the difference between the RFc and RF trials in which the saccade was to the same location but the visual display was different) and (2) internally guided (the difference between the RF and $\mathrm{nRF}$ trials in which the visual display was identical but the saccade was made to different targets). Although the external influences have been the main focus of many previous studies, the main focus of the rest of this paper will be on the modulation of visual and anticipatory responses within the internally guided context situation.

\section{Individual neuronal analysis}

Wilcoxon-Mann-Whitney (WMW) tests (Siegel and Castellan, 1988) were performed on RF versus nRF spike counts for each neuron for both the pretarget period $(-500$ to $0 \mathrm{msec}$; WMW-pre) and the post-target period (50-550 msec; WMW-post) in the FC task. $Z$ scores above 2.58 $(\mathrm{RF}>\mathrm{nRF})$ or below $-2.58(\mathrm{nRF}>\mathrm{RF})$ are significant $(p<0.01)$ (see Fig. 8).

\section{Normalized differential activity}

To characterize the response properties of individual neurons across cortical areas, normalized differential activity scores were obtained for both the pretarget period and the post-target period in the FC task using the formula $(\mathrm{RF}-\mathrm{nRF}) /(\mathrm{RF}+\mathrm{nRF})$, where each term indicates the number of action potentials during a given time window for the indicated condition.

\section{Inverse interspike interval procedure}

Figure 2 shows the inverse interspike interval procedure (1/ISI), which was used for each trial. Basically, for any two consecutive spikes the value of interspike interval (ISI) was assigned to the time period of the first spike and to every time period between the two spikes. The spike times were measured at the millisecond level so that interval functions with 1 msec bins were created. For each trial, ISI plots were created that overlapped the preceding and following trials and truncated to a fixed time range of $2200 \mathrm{msec}$ before target onset to $2800 \mathrm{msec}$ after target onset. We took the inverse of each ISI (1/ISI) to create spike-frequency functions (Fig. 2B,C, gray lines). In this way, each trial had an activity score at every $1 \mathrm{msec}$ interval with values of $>0$ and $\leq 1 \mathrm{kHz}$. To reduce noise, the 1/ISI of each trial was condensed into $5 \mathrm{msec}$ bins by taking the mean of five $1 \mathrm{msec}$ bins of the 1/ISI at nonoverlapping $5 \mathrm{msec}$ intervals (Fig. 2B,C, black circles). We avoided smoothing and waveform convoluting to preserve independence of measures within each trial, across time. These types of data fit quite well with the assumptions of the nonparametric WMW test (Siegel and Castellan, 1988).

\section{Onset of modulation}

To determine when the activity of each neuron started to differentiate between RF and nRF trials, the WMW test was performed on the
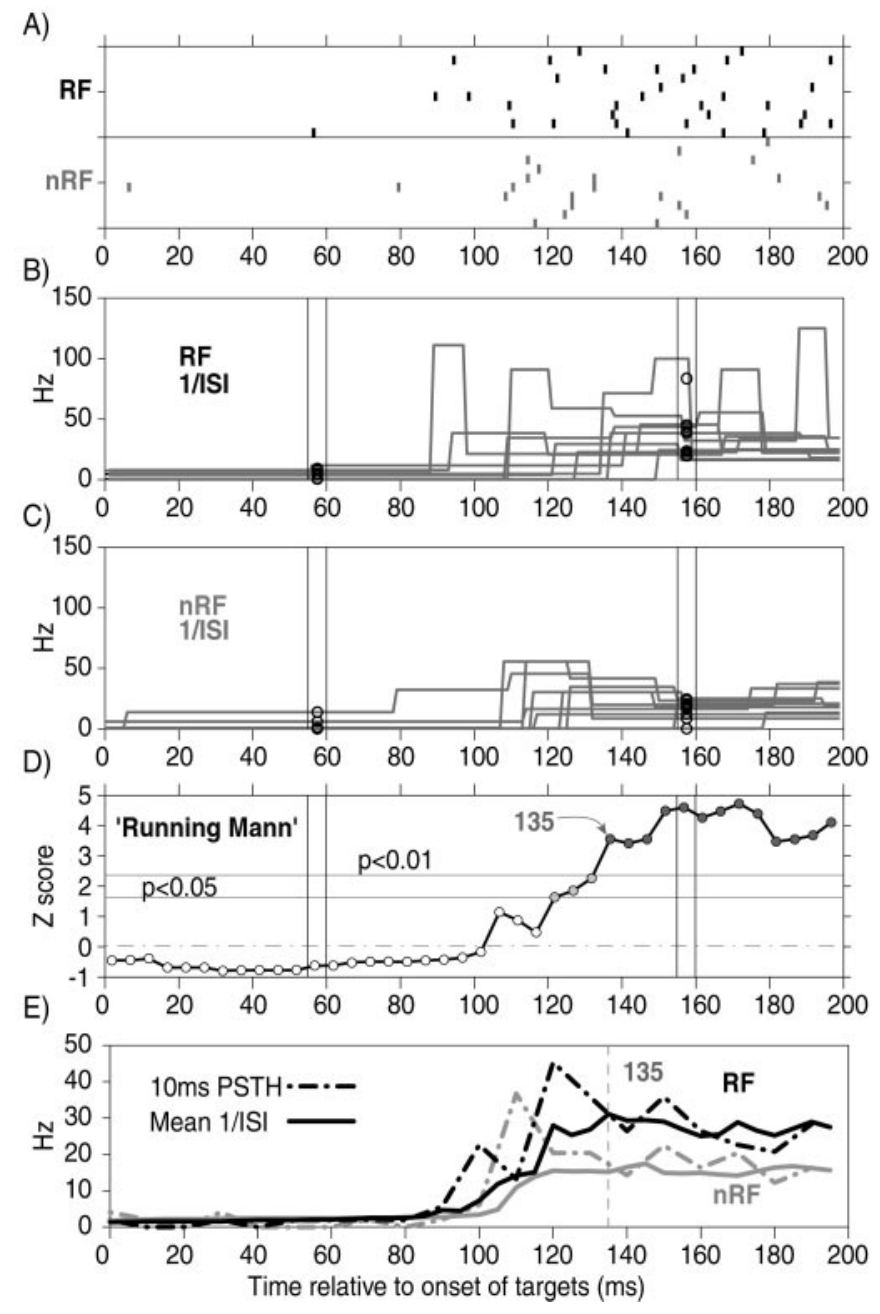

Figure 2. Explanation of the 1/ISI procedure and the Running Mann comparison. Sample data were taken from the cell in Figure $4 C . A$, Rasters for $10 \mathrm{RF}$ and $10 \mathrm{nRF}$ trials. Ticks indicate neuronal spikes. $B$, 1/ISI data for individual RF trials from the raster above are superimposed. The $5 \mathrm{msec}$ condensing process is shown at two sample time points. Each circle indicates the mean value for the 1/ISI data for each trial within the $5 \mathrm{msec}$ time window. This was done at nonoverlapping 5 msec intervals for every trial in each block. $C$, The same as $B$ but for the nRF trials from the raster above. $D$, The $Z$ scores from the running WMW tests between the two groups of condensed 1/ISI data from all of the RF and $\mathrm{nRF}$ trials ( $\sim 100$ trials). The arrow indicates the onset of the differentiation of neuronal activity, which was defined as the first of at least 10 consecutive $Z$ scores of $>2.33(p<0.01)$. $E$, The $5 \mathrm{msec}$ condensed data for all of the RF and nRF trials were then averaged across trials to form mean spike-frequency functions (black and gray solid lines, respectively). For comparison, the $10 \mathrm{msec}$ bin peristimulus time histograms of the same data are plotted with dotted lines. The vertical dotted line indicates the onset of the differentiation of neuronal activity. See Materials and Methods for details.

condensed 1/ISI spike-frequency functions. For each $5 \mathrm{msec}$ epoch, 1/ISI values from the RF trials and nRF trials were ranked and compared using the WMW test. This "Running Mann" comparison would return a $Z$ score for each $5 \mathrm{msec}$ epoch (Fig. $2 D$ ). The first of 10 consecutive $Z$ scores (i.e., $50 \mathrm{msec}$ duration) to be $>2.33(p<0.01)$ was chosen as the onset of preferential modulation (Fig. $2 E$, vertical dotted line).

\section{Mean spike-frequency function}

The mean spike-frequency function was obtained to visualize the mean changes in spike frequency by simply averaging the condensed $1 /$ ISIs for all trials in one condition (Fig. 2E). We used this method (hereafter called "1/ISI plot") to present the activities of individual neurons (Figs. 

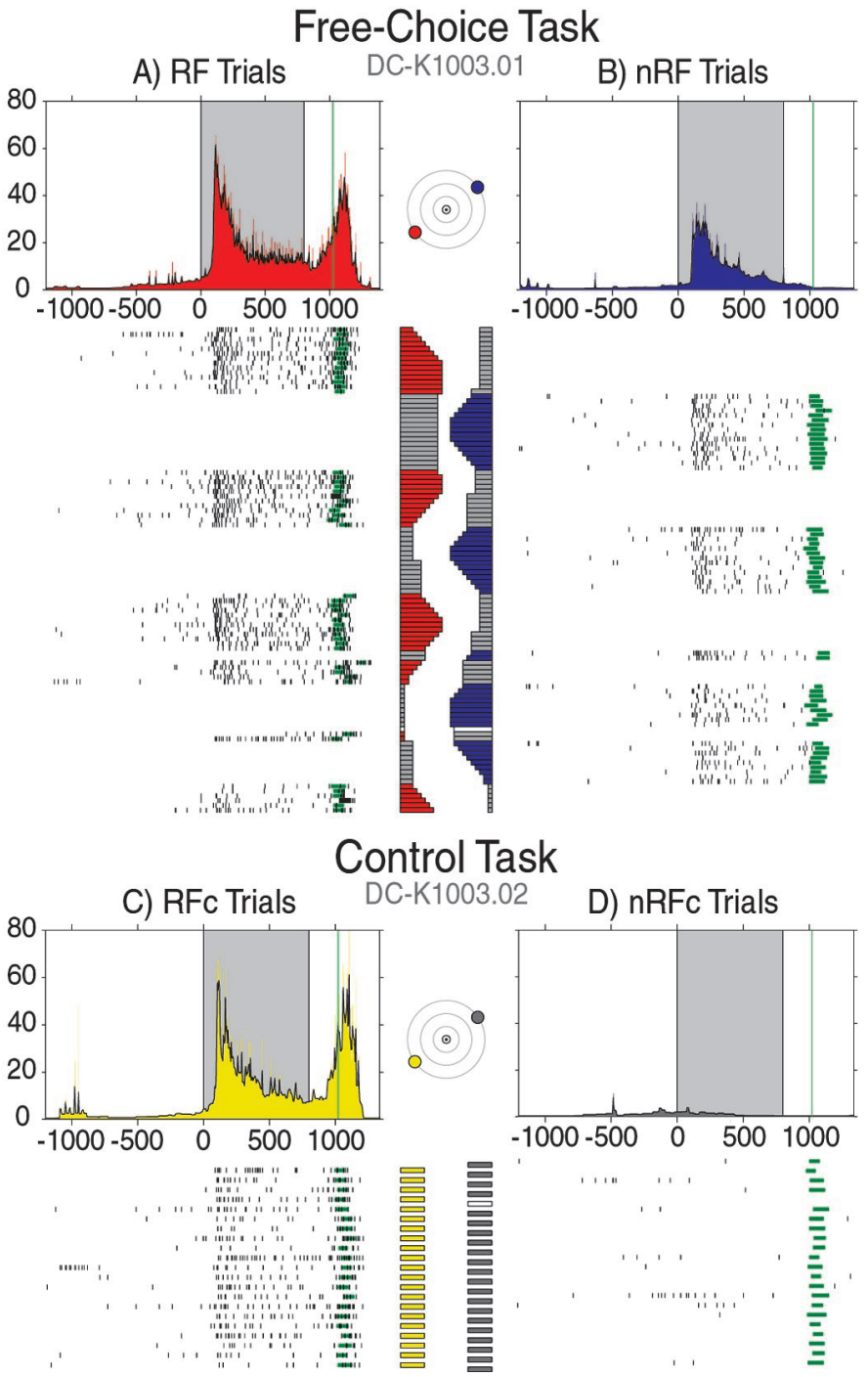

Figure 3. Visual and motor responses of a typical cell in the right FEF during the FC task (top) and the control task (bottom). Plots are arranged by task and destination of saccade. For both tasks, the activities are shown separately for both kinds of trials where a saccade was made either to the target in the RF (RF target; left-down, $30^{\circ}$ ) or to the target outside the RF (nRF target; right-up, $30^{\circ}$ ). Rasters are in chronological order from top to bottom. Black ticks indicate neuronal spikes, and green bars indicate saccade onset and duration. One millisecond 1 /ISI functions are directly above the rasters they represent and are color coded to match the target plots for each task. The $5 \mathrm{msec}$ condensed 1/ISI, or 1/ISI plots, for each are overlaid in black. The shaded area in each plot indicates the visual period $(0-800 \mathrm{msec})$, and the green line represents the mean saccade onset. The horizontal bar plots next to each raster indicate the percentage of reward for each trial. Colored sections represent the percentage of reward given for a saccade to the corresponding target. For the FC task, dark gray bars indicate amount of reward the monkey voluntarily skipped, and white bars indicate error trials in both tasks.

3-5 ) and of a population of neurons (Fig. 6). This is basically equivalent to the peristimulus time-binned histogram that has been widely used (shown in Fig. $2 E$ for comparison). The 1/ISI plot is smoother and has better temporal resolution than the $10 \mathrm{msec}$ binned histograms used in the past, while still maintaining independence of measures across time.

\section{RESULTS}

In the FC task, the monkeys switched between the two targets after several trials, displaying the behavioral pattern we were hoping for. As illustrated in Figure 3, the monkeys tended to
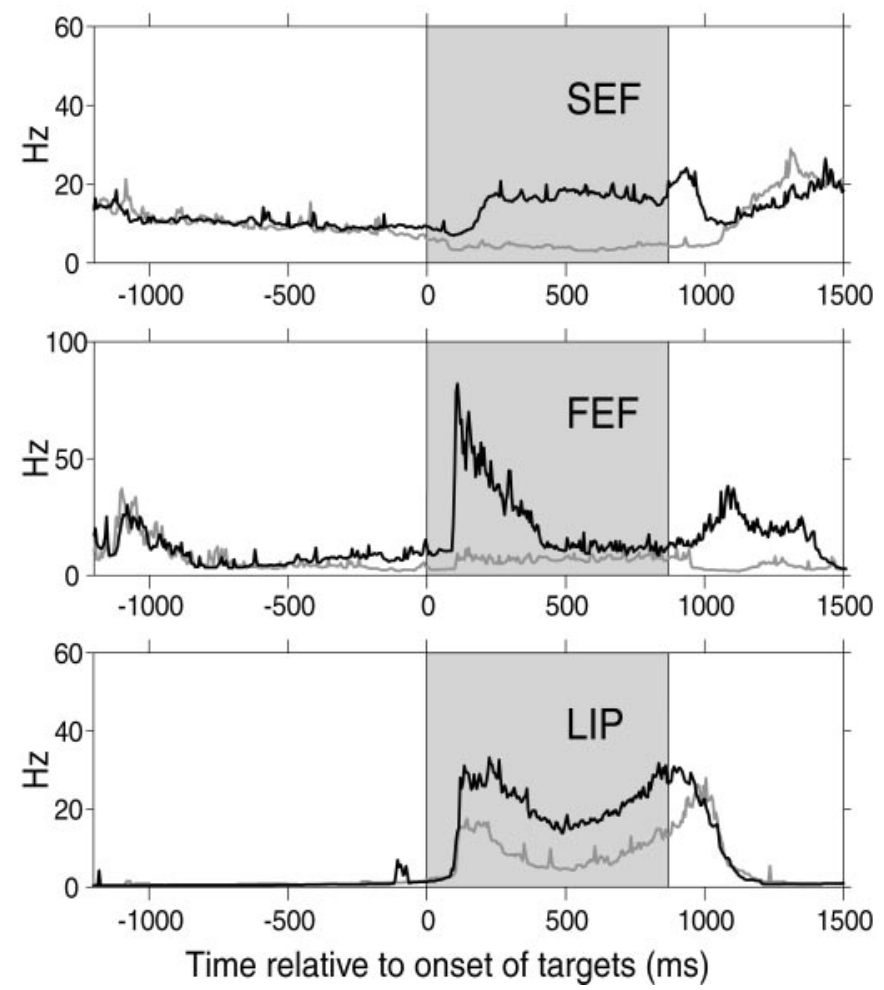

Figure 4. Strong modulation of visual response in three cortical eye fields. For a representative cell in each area, the 1/ISI plot is shown for the RF trials (black) and the nRF trials (gray) in the FC task. The difference in cell activity between the RF trials and the nRF trials is clearest during the visual period (shaded area).

choose a target for consecutive trials while the amount of reward increased, peaked, and began to decrease. Eventually the monkeys would switch to the other target, apparently seeking the more rewarding target. However, this behavior was not invariable, because the monkeys often switched between the targets after only a few trials, leaving the target with a higher percentage of reward in favor of the less rewarding target. The important point here is that the monkeys chose a target based on their own internal decision-making process and not based solely on the actual amount of reward. How the monkeys decided to which target they would saccade is an important behavioral issue that we will examine in future studies. Our main interest in this study was the modulation of visual and anticipatory activity relating to the selection of a target. Specifically, if the subject has already decided to select a given target in the FC task, attention may be allocated to that location even before the onset of the target. Accordingly, the magnitude of the early visual response and even pretarget anticipatory activity in many cells was strongly correlated with the monkey's subsequent selection. The question then becomes "when does the neural activity start to predict the behavior?"

The activity of a typical FEF cell is shown in Figure 3. In the control task, the target was presented in the lower-left or upperright field in alternation. The FEF cell exhibited a visual and motor response to the target in the lower-left field (Fig. 3, yellow plot) but not to the target in the upper-right field (Fig. 3, gray plot). Thus, the target in the lower-left target was in the RF of the cell (RF target), whereas the target in the upper-right target was outside the RF of the cell (nRF target). These task trials were designated as the RFc and $\mathrm{nRF}$ trials, respectively. 

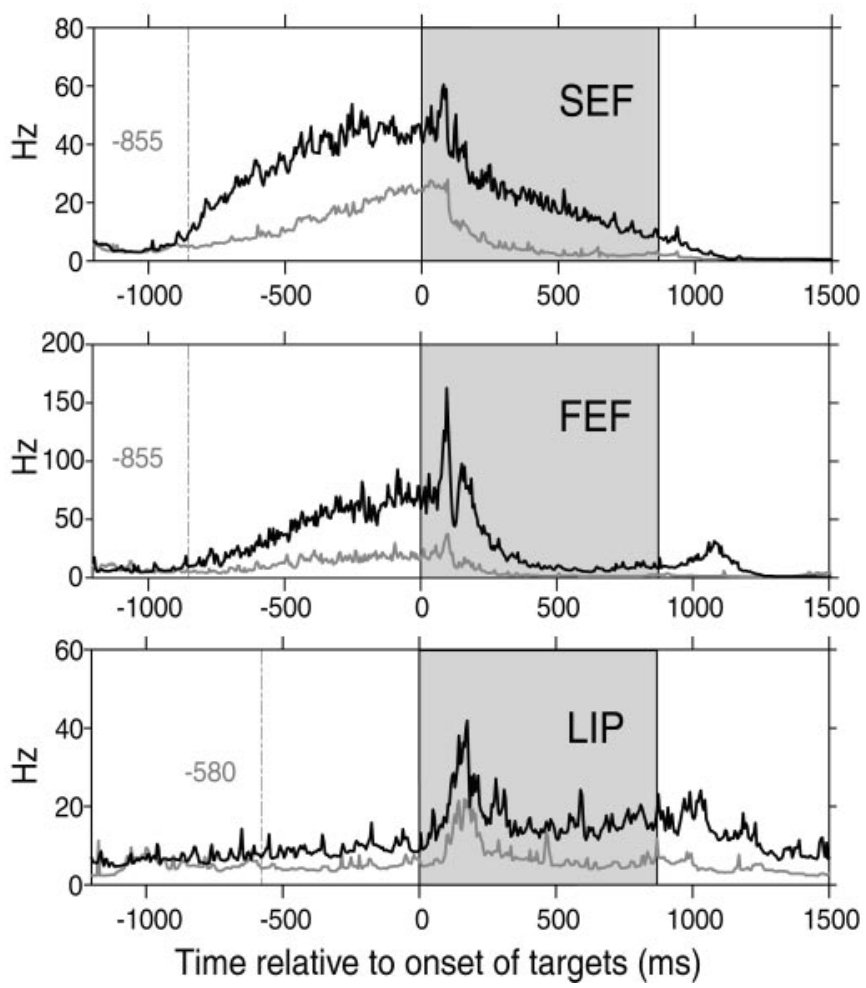

Figure 5. Strong modulation of anticipatory activity in three cortical eye fields. For a representative cell in each area, the 1/ISI plot is shown for the RF trials (black) and the nRF trials (gray) in the FC task. The difference in cell activity between the RF trials and the nRF trials is clear in the fixation period, before the onset of the targets. The dotted line in each 1/ISI plot indicates the time when the activities for the RF trials and for the nRF trials first displayed a significant difference according to the Running Mann comparison (see Materials and Methods). The shaded area indicates the visual period.

In the FC task (Fig. 3, left), the two targets were presented simultaneously and the monkey could choose either one to receive reward. The visual response of the cell was weaker (WMWpost; $p<0.01$ ) when the monkey was going to make a saccade to the nRF target (Fig. 3, blue plot) than when the monkey was going to make a saccade to the RF target (Fig. 3, red plot). These task trials were designated as the RF and nRF trials, respectively. The difference in activity between the RF and nRF trials was entirely dependent on the monkey's decision, because the presentation of stimuli was identical throughout the block of trials and both targets were associated with the full range of reward amounts. When we compare between the tasks, we can see that the neural activities during the RF trials and RFc trials were quite similar (WMW-post; $p>0.10$ ). Thus the difference within the FC task would be caused by an attenuated visual response during the nRF trials.

Figure 4 shows examples of strong modulation of visual response during the FC task in cells from the SEF, FEF, and LIP. All three cells showed a striking difference in the visual response between the RF trials and the nRF trials (WMW-post; $p<0.01$ ), although the visual display was identical. In the SEF and FEF examples, the inhibition in the nRF trials was strong enough to block out almost the entire visual response.

With closer inspection of Figure 4, the examples from SEF and FEF also seem to show anticipatory modulation even before the visual response of each cell. In Figure 5 we show three different
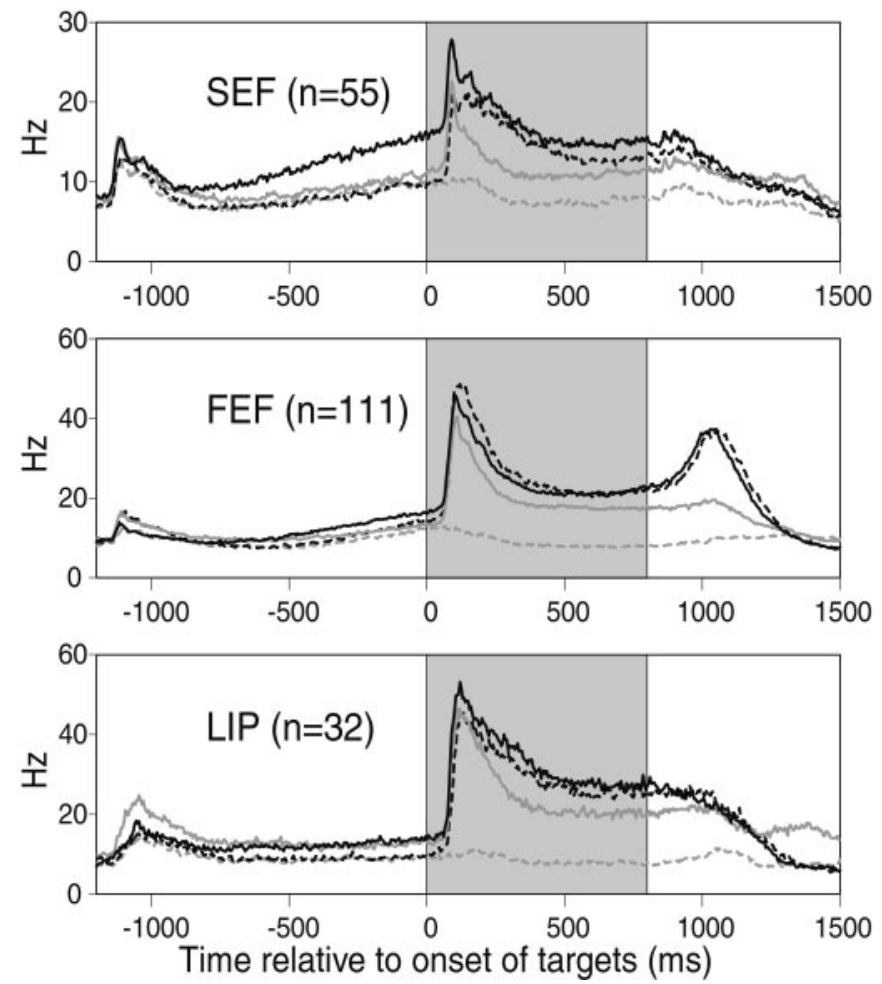

Figure 6. Population plots for three cortical eye fields. For the three populations of cells, the mean 1/ISI plots are shown for the RF trials (solid black lines) and the nRF trials (solid gray lines) of the FC task and, for comparison, the RFc trials (dotted black lines) and the $\mathrm{nRFc}$ trials (dotted gray lines) of the control task. The population data are based on 55 cells in the SEF, 111 cells in FEF, and 32 cells in LIP from five monkeys. The shaded area indicates the visual period.

cells from the SEF, FEF, and LIP with robustly enhanced anticipatory activity during the RF trials (WMW-pre; $p<0.01$ ). All three of these cells showed a striking increase of activity during the pretarget fixation period of the RF trials, although the visual targets had not yet appeared. Note that whether a given trial would be an RF trial or an nRF trial solely depended on the monkey's behavior. Thus, the anticipatory modulation of the activity of the cells reflects the monkey's decision to choose the RF target well in advance of the arrival of the visual target that it will use to execute its selection. A comparison of the cells shown in Figures 4 and 5 suggests that the monkey's decision is represented more robustly in the SEF and FEF than in the LIP.

This impression was supported by the population data (Fig. 6), which indicate that one of the clearest differences between the three cortical areas was before the onset of the targets (white portion on the left of each plot). Cells in the SEF showed the strongest modulation of anticipatory activity during the pretarget period. The difference in anticipatory activity was less clear for the FEF and was slight for the LIP. The activity is higher in the $\mathrm{RF}$ trials than in the nRF trials in the FC task as well as both conditions of the control task (RFc and nRFc trials). This suggests that the stronger anticipatory activity in the RF trials was attributable to an enhancement of the default anticipatory activity. Because the timing of all target onsets was fixed and the targets in the control task were presented in alternation, the monkeys could, in principle, anticipate the onset of each target; however, we found no indication in the data to suggest that the monkeys anticipated a specific target in the control task. 

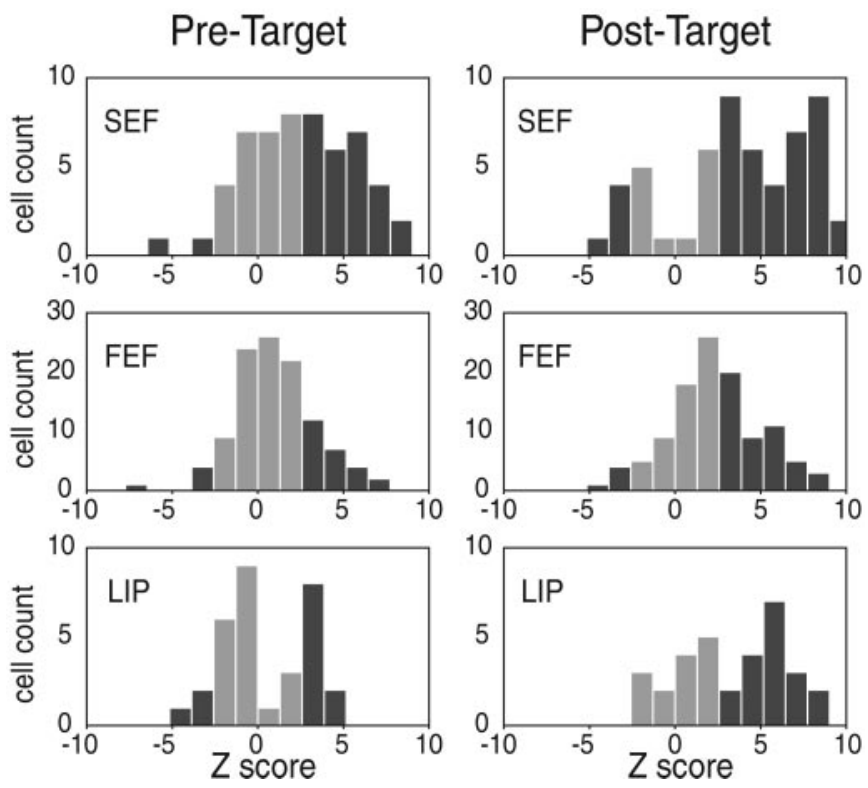

Figure 7. Distribution of $Z$ scores for pretarget and post-target activity. The spike counts for the RF trials and the nRF trials were compared using the WMW test for two separate time windows. Histograms of the results indicate how many cells showed significantly different activity. Black bars indicate cells with $Z$ scores above $2.58(\mathrm{RF}>\mathrm{nRF})$ or below -2.58 (nRF $>$ RF; $p<0.01$ ). Gray bars indicate cells with nonsignificant $Z$ scores. Left, Pretarget time window: $49.1 \%$ (27 of 55) of SEF cells, $22.5 \%$ ( 25 of 111) of FEF cells, and 31.3\% (10 of 32) of LIP cells show greater activity during the RF trials than during the nRF trials. Right, Post-target window: $67.3 \%$ (37 of 55) of SEF, $43.2 \%$ (48 of 111) of FEF, and $56.3 \%$ (18 of 32) of LIP cells show greater activity during the RF trials than during the nRF trials.

To determine whether the activity of each neuron during the RF trials was statistically different from the nRF trials, WMW tests were performed on the spike count during $500 \mathrm{msec}$ of the pretarget and post-target periods (Fig. 7). During the post-target period, $67.3 \%$ (37 of 55) of SEF neurons, $43.2 \%$ (48 of 111) of FEF neurons, and $56.3 \%$ (18 of 32) of LIP neurons displayed significantly stronger activity during the RF trials than during the nRF trials. During the pretarget period, 49.1\% (27 of 55) of SEF neurons, $22.5 \%$ (25 of 111) of FEF neurons, and 31.3\% (10 of 32) of LIP neurons displayed significantly stronger activity during the RF trials than during the nRF trials. During both time windows, a remarkable percentage of cells from all areas, especially in the SEF, seemed to reflect the monkey's intention (i.e., as to which target it had decided to select). However, the difference between the SEF and the other two eye fields seems greater during the pretarget period than during the post-target period.

To characterize the response properties of the individual neurons for each area, pretarget and post-target normalized differential activity scores were calculated for each neuron (Fig. 8). Scores could vary from 1 to -1 and a positive score indicates that the neuron showed stronger activity during the RF trials than during the nRF trials. We used this methodology to help visualize the variation within groups and for statistical analysis between groups. During the post-target period, SEF neurons had significantly higher scores than FEF neurons $(p<0.01 ; t$ test) and showed a strong tendency for higher scores than LIP neurons ( $p=0.052 ; t$ test). Neurons from the FEF and LIP had scores that were quite similar. During the pretarget period, SEF neurons had significantly higher scores than both FEF and LIP neurons
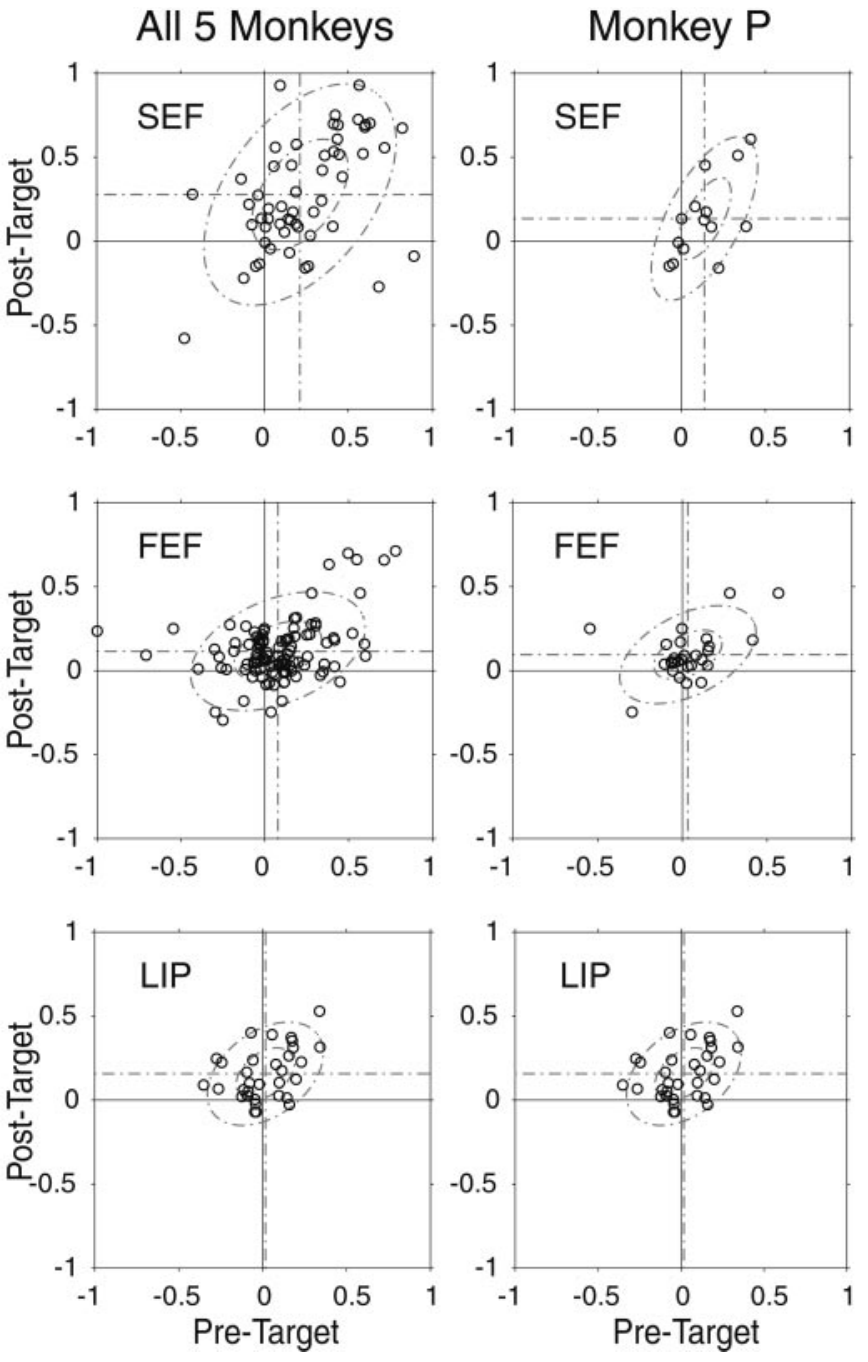

Figure 8. Normalized differential activity plots for three cortical eye fields of all five monkeys (left) and monkey $\mathrm{P}$ (right). Positive values indicate greater activity during the RF trials than during the nRF trials. Each circle represents a single neuron. In each plot, horizontal dotted lines represent the mean score for the post-target time window, and vertical dotted lines represent the mean score for the pretarget time window. The small and large ellipses indicate the first and second SD of the bivariate normal distribution.

( $p<0.01 ; t$ test) and again, neurons from the FEF and LIP had scores that were quite similar. In Figure 8, we show the pretarget scores plotted against the post-target scores to demonstrate correlations. The SEF shows stronger Pearson correlation coefficients between pretarget and post-target normalized differential activity scores than do the FEF and LIP (SEF, $r=0.483, p<$ 0.01 ; FEF, $r=0.395, p<0.01$; LIP, $r=0.399, p<0.05)$. The small and large ellipses show the first and second SD for the bivariate normal approximation, respectively. Thus, despite the variation in activity, visually responsive cells in the SEF encode the intention of the subject to select the RF target earlier than visually responsive cells in either the FEF or LIP, and modulation in the post-target period is more often preceded by modulation in the pretarget period of visually responsive cells in the SEF than visually responsive cells in either the FEF or LIP.

Finally, we compared the normalized differential activity scores for both time windows from the FEF and SEF of monkey P, from whom we collected data from all three areas, 


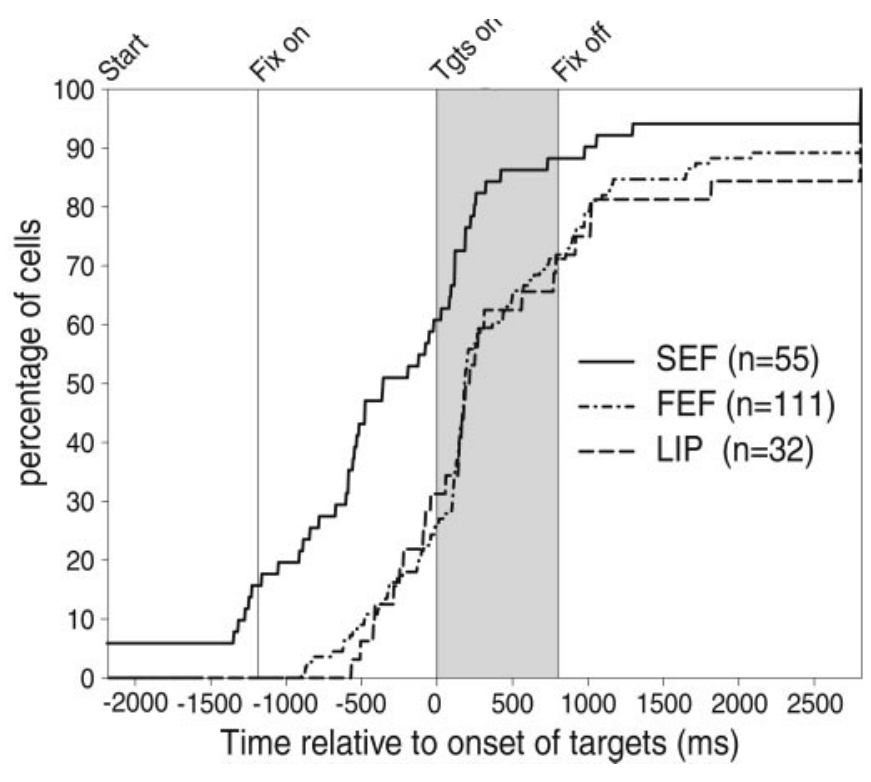

Figure 9. The onset of modulation predicting the monkey's decision to choose the RF target in three cortical eye fields. Cumulative histograms for each cortical area indicate when individual cells started showing preferential activity for the RF trials over the $\mathrm{nRF}$ trials as determined by the Running Mann comparison. The shaded area indicates the visual period and the vertical line indicates fixation onset. The majority of visually responsive cells from all three areas showed modulation before the offset of the fixation point $(800 \mathrm{msec})$. Although some cells in the FEF and LIP $(\sim 30 \%)$ showed modulation before fixation onset, this type of modulation was more prevalent in cells in the SEF (58.2\%). Three cells in the SEF showed predictive differentiated activation throughout the intertrial interval (indicated by the initial elevation). All three areas had some cells that never showed preferential activity for the RF trials in the FC task even after making a saccade (indicated by the final elevation on the right). Tgts, Targets.

with scores from the FEF and SEF of the other four monkeys to check for reliability across subjects. $t$ tests revealed no significant differences.

To further test the hypothesis that the monkey's decision is represented differently and at different times in the three cortical areas, we attempted to measure when the activity of a cell started to indicate the monkey's decision (i.e., make a saccade to the RF target). For this purpose we performed a running WMW test on the inverse interspike intervals (see Materials and Methods). The results from this Running Mann comparison on the individual examples shown in Figure 5 indicate that the onset of decision-related modulation was 855 msec before the onset of the targets for both the SEF cell and the FEF cell and $580 \mathrm{msec}$ for the LIP cell. In this way, we determined the onset of decision-related modulation for every cell. The results for all cells are plotted in Figure 9 as a cumulative histogram for each cortical area. The onset of decision-related modulation was clearly earlier for the SEF population than for the FEF or LIP populations (WMW test; $p<0.01)$; there was no significant difference between the FEF and LIP. In the SEF, 58.2\% of the cells started reflecting the monkey's decision before the target onset period, whereas only $27 \%$ of the cells in the FEF and $34.4 \%$ of the cells in the LIP did so. A few cells in the SEF $(5.5 \%)$ had a higher resting level, reflecting the monkey's decision even before the trial started. This was not found in the FEF or LIP.

\section{DISCUSSION Mechanisms of internally guided decision making
for saccades}

An important conclusion of this study is that the SEF rather than the FEF or LIP plays a leading role in the internally guided decision-making mechanisms for saccade generation. In the FC task, the subject generally chose the same target for several trials in a row and most likely had already decided the direction in which the subject was going to make a saccade well before the targets appeared. Accordingly, we found many neurons in the cortical eye fields that started to change their firing rate depending on the monkey's decision even before target presentation (Figs. 5 and 6). This anticipatory bias reflects an internal decisionmaking process. What was striking in our findings was that the anticipatory bias occurred earlier and was more common in the SEF than in the FEF or LIP. The results suggest that anticipatory bias is generated either in the SEF or in a region more directly linked to the SEF than the FEF or LIP. The bias signals in the SEF might then be transmitted to the FEF and LIP through their well known connections (Huerta and Kaas, 1990; Tian and Lynch, 1996).

Neurons from these three cortical eye fields have been shown to have similar visual or saccadic motor properties (Barash et al., 1991a; Schall, 1991a; Russo and Bruce, 2000). However, other studies suggest that the SEF has some unique, more cognitive properties: object-centered coding of visual stimuli (Olson and Gettner, 1995; Olson and Tremblay, 2000), saccades evoked by microstimulation that vary with eye position (Schall, 1991b), saccades evoked in the SEF that can be goal-directed (Fujii et al., 1995), learning of arbitrary visuomotor associations (Chen and Wise, 1995a,b, 1996), enhanced activities in conflict situations (Schlag-Rey et al., 1997), reward-related activities (Amador et al., 2000), performance monitoring (Stuphorn et al., 2000), and coding of learned visuomotor sequences ( $\mathrm{Lu}$ et al., 2002). These results suggest that aspects of previous sensory signals are first stored in the SEF and later used for movement initiation; this would be a key feature of internally guided decision making.

The hypothesized function of the SEF in internally guided decision making might be related to its location in the dorsomedial frontal cortex. It has been reported that patients with lesions in the supplementary motor area (SMA) may show a loss of spontaneous motor activity (Laplane et al., 1977). Clinical findings led to the suggestion that the SMA plays a role in decision making based on internal drives (Goldberg, 1985). This hypothesis appears to be supported by subsequent experimental studies showing that memory for which target button was rewarded was more important for success than the visual aspects of the task (Kurata and Wise, 1988). In addition, SMA neurons may be related to a long-lasting process leading to initiation of self-paced movement (Okano and Tanji, 1987; Tanji and Shima, 1994) and, in conjunction with the basal ganglia, part of a distributed neuronal system for movement initiation (Romo and Schultz, 1987; Hikosaka et al., 2000). The anterior part of the SMA, which is now called the pre-SMA, may also be related to decision making. Pre-SMA neurons may become active when the monkey changes its behavior in response to a sensory signal (Shima et al., 1996) or starts learning a new procedure (Nakamura et al., 1998). Imaging studies in humans have also indicated a role for the pre-SMA in the internal selection of movement (Deiber et al., 1996; Jenkins et al., 2000). Particularly remarkable are findings in the anterior cingulate cortex, which is just ventral to the SMA (for review, see 
Paus, 2001). Many anterior cingulate neurons became active when the monkey changed its behavior in response to a large reduction of the amount of reward (Shima and Tanji, 1998) or when the monkey found a correct answer by inference (Procyk et al., 2000). Anatomical studies also indicate that these structures are interconnected (Huerta and Kaas, 1990). Together, these results suggest that the medial frontal cortex, including the anterior cingulate cortex, pre-SMA, and SEF, may form a functional network that uses contextual clues and internal biases to select appropriate actions.

\section{Relationship between decision making and voluntary attention}

In our FC task, we found that the phasic and sustained visual responses of many neurons were stronger when the monkey was going to make a saccade to the RF target (RF trials) than to the nRF target (nRF trials) (Figs. 4 and 6). Because the target presentation was identical in both situations, the modulation of neuronal activity was solely attributable to the monkey's intention to select the RF target. Similar results have been described using an interesting paradigm where the monkeys freely scanned still images. Some FEF neurons showed a stronger visual response before saccades to a location within the RF of the neuron than before saccades to a location outside the RF of the neuron (Burman and Segraves, 1994). The authors argue that the activity of FEF neurons seemed to depend on the monkey's level of arousal or engagement of attention. The modulation of visual response may include two kinds of processes: voluntary attention (Bushnell et al., 1981; Kodaka et al., 1997; Luck et al., 1997) and motor preparation (Goldberg and Bushnell, 1981). For the FEF and SEF cells in Figure 4, this visual modulation, or bias, was sometimes so strong that the response to the RF stimulus was eliminated when the monkey was going to choose the nRF stimulus. In this situation, top-down inhibitory modulation completely countermanded the bottom-up visual response of the neuron.

In the FC task, the monkeys had already decided the direction in which they were going to saccade by the time the targets appeared and could allocate attention to a specific location in space. This biased the neural response even before the onset of the targets. For many SEF cells, this anticipatory bias was correlated with visual modulation. Similar findings have been discussed for V4 neurons, for which the modulation appeared before the target onset. However, this modulation took place after a visual response to the onset of two location-marking frames (Luck et al., 1997).

One possibility for the origin of the visual bias is that the strong anticipatory bias in the SEF (Fig. 6) might be transmitted to the FEF and LIP (Huerta and Kaas, 1990), which, together with visual inputs, would initiate the visual biases in the FEF and LIP. This initial visual bias might then be enhanced and maintained if the visual signals are circulated through the three cortical eye fields (Stanton et al., 1993, 1995; Tian and Lynch, 1996; Chafee and Goldman-Rakic, 2000).

\section{Relationship between externally guided and internally guided decision making}

There have been many studies on the neural mechanisms of externally guided decision making (Shadlen et al., 1996; Thompson et al., 1996; Schall and Bichot, 1998; Thompson and Schall, 1999). In these studies, the subject would make a perceptual decision by detecting the presence of or a characteristic of a single stimulus or by discriminating between stimuli presented; the subject would then perform a fixed behavioral response. The subject's decision is then directly linked to, or the result of, immediate sensory evaluation. In a typical case, the physical features of the sensory stimulus, or a cueing stimulus, determine the subject's behavior, because the subject has been taught as such. This type of selection behavior may be predicted mathematically from neuronal activity (Gold and Shadlen, 2001).

However, primates are thought to behave based on some internal factors in addition to external factors. These internal factors are ill defined and can include expectation, inference, preference, mood, habits, fatigue, and guessing. For this reason, even the most basic discrimination task must still involve some internal factors. Likewise, no task could be exclusively internally driven, because external factors such as visual display, auditory events, and timing instruct the subject what needs to be done and when. In this paper, the terms internal and external are used for instructional purposes as labels for situations where the majority of influences would be biased in one manner or the other. By introducing small and imprecise alterations in water release during our task, we hoped to significantly enhance the role of dynamic internal factors in a task where the rest of the external factors remained constant. In this way we gave the subject the freedom to choose the target that it expected, inferred, felt, or guessed was in some way preferable while still maintaining a controlled environment. A similar paradigm, based on a matchto-sample format, has been used on humans. In that case, the experimenter simply asked the subjects which one of three stimuli they "like the best" and compared the results with previous blocks for which the subjects were asked to select the most similar or most different (Goldberg and Podell, 2000). Using their cognitive bias task, they found perseverance-type deficits in prefrontal lobe patients that the Wisconsin card-sorting task did not elucidate, arguing that to truly investigate decision making and the frontal lobes, one needs to use more dynamic preference-based tasks.

Strong manipulation of reward has induced modulations of neuronal activities in cortical areas (Watanabe, 1996; Tremblay and Schultz, 2000) including the FEF (Kobayashi et al., 2002), area 46 but not the FEF (Leon and Shadlen, 1999), the LIP (Platt and Glimcher, 1999), the supplementary motor cortex (Kurata and Wise, 1988), and basal ganglia nuclei (Bowman et al., 1996; Kawagoe et al., 1998; Schultz, 1998; Shimo and Hikosaka, 2001). However, most of the reward-related modulations occurred after presentation of targets or instructional cues that explicitly instructed the subject. Notable exceptions have been found recently in the caudate (Lauwereyns et al., 2002; Takikawa et al., 2002), the substantia nigra pars reticulata (Sato and Hikosaka, 2000), and the SC (Ikeda et al., 2001), all of which showed pretarget activity that depended on the context of an asymmetric reward schedule that remained fixed throughout each block.

These data suggest that different neural networks are involved in different aspects of decision making. We believe that by directly comparing three cortical eye fields, the present results provide an important development in the study of neural mechanisms for both internally guided and externally guided decisionmaking mechanisms.

\section{REFERENCES}

Amador N, Schlag-Rey M, Schlag J (2000) Reward-predicting and reward-detecting neuronal activity in the primate supplementary eye field. J Neurophysiol 84:2166-2170.

Andersen RA, Gnadt JW (1989) Posterior parietal cortex. In: The neurobiology of saccadic eye movements (Wurtz RH, Goldberg ME, eds), pp 315-335. Amsterdam: Elsevier. 
Barash S, Bracewell RM, Fogassi L, Gnadt JW, Andersen RA (1991a) Saccade-related activity in the lateral intraparietal area. I. Temporal properties; comparison with area 7a. J Neurophysiol 66:1095-1108.

Barash S, Bracewell RM, Fogassi L, Gnadt JW, Andersen RA (1991b) Saccade-related activity in the lateral intraparietal area. II. Spatial properties. J Neurophysiol 66:1109-1124.

Bowman EM, Aigner TG, Richmond BJ (1996) Neural signals in the monkey ventral striatum related to motivation for juice and cocaine rewards. J Neurophysiol 75:1061-1073.

Bruce CJ, Goldberg ME (1985) Primate frontal eye fields. I. Single neurons discharging before saccades. J Neurophysiol 53:603-635.

Bruce CJ, Goldberg ME, Bushnell MC, Stanton GB (1985) Primate frontal eye fields. II. Physiological and anatomical correlates of electrically evoked eye movements. J Neurophysiol 54:714-734.

Burman DD, Segraves MA (1994) Primate frontal eye field activity during natural scanning eye movements. J Neurophysiol 71:1266-1271.

Bushnell MC, Goldberg ME, Robinson DL (1981) Behavioral responses in monkey cerebral cortex. I. Modulation in posterior parietal cortex related to selective visual attention. J Neurophysiol 46:755-772.

Chafee MV, Goldman-Rakic PS (2000) Inactivation of parietal and prefrontal cortex reveals interdependence of neural activity during memory-guided saccades. J Neurophysiol 83:1550-1566.

Chen LL, Wise SP (1995a) Neuronal activity in the supplementary eye field during acquisition of conditional oculomotor associations. J Neurophysiol 73:1101-1121.

Chen LL, Wise SP (1995b) Supplementary eye field contrasted with the frontal eye field during acquisition of conditional oculomotor associations. J Neurophysiol 73:1122-1134.

Chen LL, Wise SP (1996) Evolution of directional preferences in the supplementary eye field during acquisition of conditional oculomotor associations. J Neurosci 16:3067-3081.

Coe B, Tomihara K, Hikosaka O (1998) Activity of visually responsive frontal eye field neurons modulated by intention. Soc Neurosci Abstr 24:659.7.

Coe B, Matsuzawa M, Hikosaka O (2001) Visual and anticipatory modulation in FEF SEF, LIP of the monkey in a free-choice task. Soc Neurosci Abstr 27:533.15.

Colby CL, Duhamel J-R, Goldberg ME (1996) Visual, presaccadic, and cognitive activation of single neurons in monkey lateral intraparietal area. J Neurophysiol 76:2841-2852.

Deiber MP, Ibanez V, Sadato N, Hallett M (1996) Cerebral structures participating in motor preparation in humans: a positron emission tomography study. J Neurophysiol 75:233-247.

Fujii N, Mushiake H, Tamai M, Tanji J (1995) Microstimulation of the supplementary eye field during saccade preparation. NeuroReport 6:2565-2568.

Gold JI, Shadlen MN (2001) Neural computations that underlie decisions about sensory stimuli. Trends Cogn Sci 5:10-16.

Goldberg E, Podell K (2000) Adaptive decision making, ecological validity, and the frontal lobes. J Clin Exp Neuropsychol 22:56-68.

Goldberg G (1985) Supplementary motor area structure and function: review and hypothesis. Behav Brain Sci 8:567-616.

Goldberg ME, Bushnell MC (1981) Behavioral enhancement of visual responses in monkey cerebral cortex. II. Modulation in frontal eye fields specifically related to saccades. J Neurophysiol 46:773-787.

Hikosaka O, Wurtz RH (1983) Visual and oculomotor functions of monkey substantia nigra pars reticulata. III. Memory-contingent visual and saccade responses. J Neurophysiol 49:1268-1284.

Hikosaka O, Takikawa Y, Kawagoe R (2000) Role of the basal ganglia in the control of purposive saccadic eye movements. Physiol Rev 80:953-978.

Huerta MF, Kaas JH (1990) Supplementary eye field as defined by intracortical microstimulation: connections in macaques. J Comp Neurol 293:299-330.

Ikeda T, Takikawa Y, Hikosaka O (2001) Visuo-motor and anticipatory activities of monkey superior colliculus neurons are modulated by reward. Soc Neurosci Abstr 27:59.1.

Jenkins IH, Jahanshahi M, Jueptner M, Passingham RE, Brooks DJ (2000) Self-initiated versus externally triggered movements. II. The effect of movement predictability on regional cerebral blood flow. Brain 123:1216-1228.

Kawagoe R, Takikawa Y, Hikosaka O (1998) Expectation of reward modulates cognitive signals in the basal ganglia. Nat Neurosci 1:411-416.

Kim J-N, Shadlen MN (1999) Neural correlates of a decision in the dorsolateral prefrontal cortex of the macaque. Nat Neurosci 2:176-185.

Kobayashi S, Lauwereyns J, Koizumi M, Sakagami M, Hikosaka O (2002) Influence of reward expectation on visuospatial processing in macaque lateral prefrontal cortex. J Neurophysiol 87:1488-1498.

Kodaka Y, Mikami A, Kubota K (1997) Neuronal activity in the frontal eye field of the monkey is modulated while attention is focused onto a stimulus in the peripheral visual field, irrespective of eye movement. Neurosci Res 28:291-298.

Kurata K, Wise SP (1988) Premotor and supplementary motor cortex in rhesus monkeys: neural activity during externally- and internallyinstructed motor tasks. Exp Brain Res 72:237-248.

Laplane D, Talairach J, Meiningerm V, Bancaud J, Orgogozo JM (1977) Clinical consequences of corticectomies involving the supplementary motor area in man. J Neurol Sci 34:301-314.

Lauwereyns J, Takikawa Y, Kawagoe R, Kobayashi S, Koizumi M, Coe B, Sakagami M, Hikosaka O (2002) Feature-based anticipation of cues that predict reward in monkey caudate nucleus. Neuron 33:463-473.

Leon MI, Shadlen MN (1999) Effect of expected reward magnitude on the response of neurons in the dorsolateral prefrontal cortex of the macaque. Neuron 24:415-425.

Lu X, Matsuzawa M, Hikosaka O (2002) A neural correlate of oculomotor sequences in supplementary eye field. Neuron 34:317-325.

Luck S, Chelazzi L, Hillard S, Desimone R (1997) Neural mechanisms of spatial selective attention in areas V1, V2, and V4 of macaque visual cortex. J Neurophysiol 77:24-42.

Mohler CW, Goldberg ME, Wurtz RH (1973) Visual receptive fields of frontal eye field neurons. Exp Brain Res 61:385-389.

Nakamura K, Sakai K, Hikosaka O (1998) Neuronal activity in medial frontal cortex during learning of sequential procedures. J Neurophysiol 80:2671-2687.

Okano K, Tanji J (1987) Neuronal activities in the primate motor fields of the agranular frontal cortex preceding visually triggered and selfpaced movement. Exp Brain Res 66:155-166.

Olson CR, Gettner SN (1995) Object-centered direction selectivity in the macaque supplementary eye field. Science 269:985-988.

Olson CR, Tremblay L (2000) Macaque supplementary eye field neurons encode object-centered locations relative to both continuous and discontinuous objects. J Neurophysiol 83:2392-2411.

Paré M, Wurtz RH (1997) Monkey posterior parietal cortex neurons antidromically activated from superior colliculus. J Neurophysiol 78:3493-3497.

Paus T (2001) Primate anterior cingulate cortex: where motor control, drive, and cognition interface. Nat Rev Neurosci 2:417-424.

Platt ML, Glimcher PW (1999) Neural correlates of decision variables in parietal cortex. Nature 400:233-238.

Procyk E, Tanaka YL, Joseph JP (2000) Anterior cingulate activity during routine and non-routine sequential behaviors in macaques. Nat Neurosci 3:502-508.

Robinson DA (1963) A method of measuring eye movement using a scleral search coil in a magnetic field. IEEE Trans Biomed Eng 10:137-145.

Romo R, Schultz W (1987) Neuronal activity preceding self-initiated or externally timed arm movements in area 6 of monkey cortex. Exp Brain Res 67:656-662.

Russo GS, Bruce CJ (2000) Supplementary eye field: representation of saccades and relationship between neural response fields and elicited eye movements. J Neurol 84:2605-2621.

Sato M, Hikosaka O (2000) Reward-related modulation of spatial information in substantia nigra pars reticulata neurons for subsequent saccades. Soc Neurosci Abstr 26:254.11.

Schall JD (1991a) Neuronal activity related to visually guided saccadic eye movements in the supplementary motor area of rhesus monkeys. J Neurophysiol 66:530-558.

Schall JD (1991b) Neuronal activity related to visually guided saccades in the frontal eye fields of rhesus monkeys: comparison with supplementary eye fields. J Neurophysiol 66:559-579.

Schall JD (1995) Neural basis of saccade target selection. Rev Neurosci 6:63-85.

Schall JD (2001) Neural basis of deciding, choosing, and acting. Nat Neurosci 2:33-42.

Schall JD, Bichot NP (1998) Neural correlates of visual and motor decision processes. Curr Opin Neurobiol 8:211-217.

Schlag J, Schlag-Rey M (1987) Evidence for a supplementary eye field. J Neurophysiol 57:179-200.

Schlag-Rey M, Amador N, Sanchez H, Schlag J (1997) Antisaccade performance predicted by neuronal activity in the supplementary eye field. Nature 390:398-401.

Schultz W (1998) Predictive reward signal of dopamine neurons. J Neurophysiol 80:1-27.

Segraves MA, Goldberg ME (1987) Functional properties of corticotectal neurons in the monkey's frontal eye field. J Neurophysio 58:1387-1419.

Shadlen MN, Britten KH, Newsome WT, Movshon JA (1996) A computational analysis of the relationship between neuronal and behavioral responses to visual motion. J Neurosci 16:1486-1510.

Shima K, Tanji J (1998) Role for cingulate motor area cells in voluntary movement selection based on reward. Science 282:1335-1338.

Shima K, Mushiake H, Saito N, Tanji J (1996) Role for cells in the presupplementary motor area in updating motor plans. Proc Natl Acad Sci USA 93:8694-8698.

Shimo Y, Hikosaka O (2001) Role of tonically active neurons in primate caudate in reward-oriented saccadic eye movement. J Neurosci $21: 7804-7814$. 
Shook BL, Schlag-Rey M, Schlag J (1990) Primate supplementary eye field. I. Comparative aspects of mesencephalic and pontine connections. J Comp Neurol 301:618-642.

Siegel S, Castellan NJ (1988) Nonparametric statistics for the behavioral sciences. New York: McGraw-Hill.

Sommer MA, Wurtz RH (2000) Composition and topographic organization of signals sent from the frontal eye field to the superior colliculus. J Neurophysiol 83:1979-2001.

Stanton GB, Bruce CJ, Goldberg ME (1993) Topography of projections to the frontal lobe from the macaque frontal eye fields. J Comp Neurol 330:286-301.

Stanton GB, Bruce CJ, Goldberg ME (1995) Topography of projections to posterior cortical areas from the macaque frontal eye fields. J Comp Neurol 353:291-305.

Stuphorn V, Taylor TL, Schall JD (2000) Performance monitoring by the supplementary eye field. Nature 408:857-860.

Takikawa Y, Kawagoe R, Hikosaka O (2002) Reward-dependent spatial selectivity of anticipatory activity in monkey caudate neurons. J Neurophysiol 87:508-515.

Tanji J, Shima K (1994) Role for supplementary motor area cells in planning several movements ahead. Nature 371:413-416.

Thompson KG, Schall JD (1999) The detection of visual signals by macaque frontal eye field during masking. Nat Neurosci 2:283-288.

Thompson KG, Hanes DP, Bichot NP, Schall JD (1996) Perceptual and motor processing stages identified in the activity of macaque frontal eye field neurons during visual search. J Neurophysiol 76:4040-4055.

Tian J-R, Lynch JC (1996) Corticocortical input to the smooth and saccadic eye movement subregions of the frontal eye field in cebus monkeys. J Neurophysiol 76:2754-2771.

Tremblay L, Schultz W (2000) Reward-related neuronal activity during go-nogo task performance in primate orbitofrontal cortex. J Neurophysiol 83:1864-1876.

Watanabe M (1996) Reward expectancy in primate prefrontal neurons. Nature 382:629-632. 